López, Marcelo Luis (2016). Extensión universitaria y salud pública en Argentina como caso testigo en la región. Revista de Investigación Educativa, 34(1), 119-132.

DOI: http://dx.doi.org/10.6018/rie.34.1.215411

\title{
Extensión universitaria y salud pública en Argentina como caso testigo en la región"
}

\section{Public health and university extension in Argentina as witness case at the region}

\author{
Marcelo Luis López \\ Facultad de Humanidades y Ciencias Sociales \\ Universidad Nacional de Jujuy (Argentina)
}

\begin{abstract}
Resumen
El artículo es un avance de un proyecto de investigación sobre extensión universitaria (EU): función de la educación superior orientada al desarrollo social, cultural, productivo y medioambiental de los entornos universitarios. Pese a su relevancia la misma se desarrolla bastante pragmáticamente con poca orientación teórica por lo que estos estudios brindan elementos para la praxis extensionista. El objetivo del trabajo es mejorar la comprensión de la relación teoría-práctica en extensión universitaria en este caso delimitada al campo de la salud/salud pública. A partir del análisis de ponencias de congresos nacionales de extensión de Argentina, como caso testigo en la región, se examinan los resultados de cómo estos eventos están aportando a la construcción del objeto EU a partir de la red bibliográfica y el reagrupamiento de temas, objetos y sujetos. La metodología con la que se trabajó es la Grounded Theory.

Palabras clave: Argentina, responsabilidad social, educación superior, salud pública.
\end{abstract}

\footnotetext{
Correspondencia: Marcelo Luis Lopez (marllopez@yahoo.es)

* El artículo deriva del proyecto de investigación (Res. N ${ }^{\circ}$ CS 164/12 código SECTER C-B006 desarrollado en el ámbito -con subsidio- de la Secretaría de Ciencia y Técnica y Estudios Regionales de la Universidad Nacional de Jujuy de la República Argentina.
} 


\section{Summary}

The article is a preliminary research about Extensión Universitaria (EU): its function is oriented to the social, cultural, productive and environmental development in this Higher Education contexts. Even though it is important, it has been done with little theoretical orientations. Thus, these studies only provide elements for praxis. The main aim of this article is to understand the relationship between theory and practice in the EU precisely circumscribed to: Health / Public Health. The methodology used is the Grounded Theory and this research is based on the analysis of different papers presented at National Conferences of EU in Argentina, as an example in the region. Results are related to the construction of the EU object and bibliographic network, regrouping of subjects, objects and subjects are examined.

Keywords: Argentina, higher education, public health, social responsibility.

\section{Introducción}

La extensión universitaria, junto con la docencia y la investigación, en muchos ámbitos universitarios es reconocida como una de las funciones/misiones con las que el discurso de la educación superior actual constituye su identidad diferenciándose de otros niveles educativos (Bueno Campos, 2007; López, 2010). Se basa en la intervención directa de las universidades en procesos sociales, culturales, productivos y medioambientales a través de organizadores como extensión (propiamente dicha) u otros equivalentes como transferencia (García, 2015), vinculación, interacción social, proyección social, responsabilidad social, gestión cultural, desarrollo local (López, 2012). A diferencia de la extensión que efectúan otras agencias, por ejemplo en el área de la ruralidad, en la Argentina la extensión universitaria permite reafirmar los campos intelectuales integrados a la educación superior en relación al campo de poder (Bourdieu, 2002) como jurisdicción autónoma (López, 2005, 2010). Sin embargo, aun siendo un aspecto relevante -y pese la creciente multiplicación de experiencias y eventos que hicieron eclosión en las últimas dos décadas en la región- las mismas se llevan a cabo pragmáticamente, con poca investigación y orientación teórica (García Guadilla, 1996; López, 2011). Consecuentemente la extensión universitaria es una importante fuente productora de discursos en los diferentes campos de lo social.

En relación al discurso universitario y la función extensionista a fines de los 90 en Argentina comenzó un proceso de multiplicación exponencial de actividades sobre extensión universitaria a nivel local, regional, nacional e iberoamericano. Esta explosión tuvo como hechos clave la búsqueda de nuevos actores que se ocuparan de los problemas sociales a partir de la retracción del estado dentro las políticas neoliberales de la década y la Conferencia Mundial sobre Educación Superior de 1998 -y subsiguientes- que, además de involucrar temas endógenos propios del nivel superior como pertinencia, gestión y calidad, también acentuó el debate sobre el papel que puede cumplir la universidad en los grandes problemas de finesprincipios de milenio como democratización, exclusión social y medioambiente, siendo la función extensionista la herramienta privilegiada para considerarlos (López, 2012). En este trabajo se presentan avances recortados al campo de la salud con 
el objetivo específico de establecer vínculos entre el discurso acerca la salud/salud pública y el discurso universitario desde la función de extensión universitaria. La relación entre extensión-salud es un aspecto abordado en otros aportes (Marimon, 2007; Pérez Martínez, 2010; Veliz Gutiérrez, Pérez Díaz, Fernández Montenquin, Veliz Martínez y Pérez, 2011), aunque con diferente alcance debido a la tradición de Argentina en materia de extensión universitaria -que la hace especialmente representativa para la región-y donde además, no se abordan aspectos que se analizan aquí. Estas cuestiones son:

1) La red bibliográfica utilizada en la elaboración de ponencias sobre salud de los congresos nacionales de extensión universitaria como fuentes del discurso sobre extensión universitaria para establecer el soporte con que se van elaborando estos discursos y su aportación a la construcción del objeto extensión universitaria.

2) La elección de objetos, sujetos y áreas temáticas que se vienen priorizando en el discurso extensionista sobre salud en Argentina como caso testigo en la región iberoamericana.

\section{Metodología}

Se trabajó con fuentes documentales siendo las mismas los libros de resúmenes ponencias y pósters de los primeros cuatro congresos nacionales de extensión llevados a cabo en Argentina: Cuyo 1997 (Universidad Nacional de Cuyo, 1997), Mar del Plata 2006 (Universidad Nacional de Mar del Plata, 2006), Santa Fe 2009 (Universidad Nacional del Litoral, 2009) y Cuyo 2010 (Universidad Nacional de Cuyo, 2010).

Los criterios para la selección de dichas fuentes se orientaron a obtener manifestaciones del discurso sobre extensión universitaria considerando congresos, más que jornadas u otro tipo de encuentros, y de carácter nacional, antes que regional o local, bajo la premisa que estos aspectos implican mayor acreditación de los eventos, reunión de autoridades de áreas de extensión de la mayoría de las universidades del país, debates más profundos y concentración de exposiciones de más alto nivel representativas de la extensión que se realiza en las diferentes jurisdicciones. La metodología general considerada para el proyecto es la Grounded Theory (Soneira, 2007). En este anticipo se parte de un análisis cuantitativo-cualitativo en el que se identifican ponencias relevantes conducentes al muestreo teórico y a los procesos de codificación -y categorización- abierta y axial recortados a la temática de la salud quedando pendiente la codificación selectiva, y la aplicación de la estrategia de comparación constante, propios de la perspectiva metodológica de la Teoría Fundamentada en los Datos, para cuando se comparen similitudes y diferencias entre las categorías emergentes de las distintas áreas temáticas que se abordan en los congresos más allá del tema de la salud.

Debido a la contribución que ha significado la aplicación del Análisis de Redes Sociales (ARS) en las ciencias sociales, como lo muestran otros estudios educativos particularmente sobre educación superior (Maz-Machado et al 2012), se utilizó dicha lógica para el primer aspecto, o sea el abordaje de la red bibliográfica, con la aplicación 
del programa UCINET. Para el segundo aspecto-la elección de objetos y sujetos de la extensión-se trabajó con 192 ponencias que corresponden a salud, del total $(\mathrm{N}=1256)$, de acuerdo al siguiente detalle (Tabla 1):

Tabla 1

Distribución de ponencias y póster en los congreso nacionales de extensión de Argentina (1997-2010)

\begin{tabular}{lcc}
\hline \multicolumn{1}{c}{ Congreso } & Ponencias & Ponencias sobre salud \\
\hline Cuyo 1997 & 259 & 25 \\
Mar del Plata 2006 & 217 & 55 \\
Santa Fe 2009 & 387 & 63 \\
Cuyo 2010 & 393 & 49 \\
Total & 1256 & 192 \\
\hline
\end{tabular}

\section{Resultados}

El mapa de presencias y ausencias de las UU.NN y sus zonas de influencia en la construcción del discurso sobre salud a partir de la función extensionista (Tabla 2 y Figura 1) muestra que de las 47 Universidades del país 22 acusaron participación. Se destacan las ausencias de la Rioja, gran parte de la Provincia de Buenos aires y Santiago del Estero. Por otra parte el $72 \%$ de la producción fue desarrollado por las Universidades de La Plata, San Luis, Litoral, Mar del Plata, Tucumán, Buenos Aires, Entre Ríos, Cuyo y Córdoba.

Tabla 2

Contribuciones de las distintas universidades con ponencias sobre salud en los congresos nacionales de extensión universitaria de Argentina (1997-2010)

\begin{tabular}{lccccc}
\hline & \multicolumn{5}{c}{ Ponencias aportadas en cada congreso } \\
\hline Universidad (Nacional) & Cuyo 97 & MdP 06 & Sta. Fe 09 & Cuyo 10 & Totales* $^{*}$ \\
\hline La Plata & 1 & & 10 & 11 & $\mathbf{2 2}$ \\
San Luis & 2 & 8 & 5 & 4 & $\mathbf{1 9}$ \\
Litoral & 2 & 1 & 14 & 2 & $\mathbf{1 9}$ \\
Mar del Plata & 1 & 9 & 3 & 4 & $\mathbf{1 7}$ \\
Tucumán & 4 & 11 & & & $\mathbf{1 5}$ \\
Buenos Aires & & 2 & 5 & 6 & $\mathbf{1 3}$ \\
Entre Ríos & 1 & 7 & & 7 & $\mathbf{1 2}$ \\
Cuyo & 4 & & & 2 & $\mathbf{1 1}$ \\
Córdoba & & & & & $\mathbf{1 0}$ \\
Salta & 4 & 4 & 4 & & $\mathbf{8}$ \\
Rosario & & & & & 5 \\
\hline
\end{tabular}




\begin{tabular}{|c|c|c|c|c|c|}
\hline Nordeste & 1 & & 2 & 1 & 4 \\
\hline Univ. Brasileras & 3 & & & 1 & 4 \\
\hline La Pampa & & & 2 & 1 & 3 \\
\hline $\begin{array}{l}\text { Depto. Ext. Univ. } \\
\text { Hospital Italiano }\end{array}$ & & 2 & & & 2 \\
\hline Misiones & & & 1 & 1 & 2 \\
\hline San Juan Bosco & & & 2 & & 2 \\
\hline Univ. Uruguayas & & & 2 & & 2 \\
\hline Comahue & 1 & 1 & & & 2 \\
\hline Jujuy & 1 & & 1 & & 2 \\
\hline Catamarca & & & & 1 & 1 \\
\hline $\begin{array}{l}\text { Centro de la Prov. de } \\
\text { Bs. As. }\end{array}$ & & 1 & & & 1 \\
\hline Río Cuarto & & & & 1 & 1 \\
\hline Del Sur & & & & 1 & 1 \\
\hline San Juan & & & 1 & & 1 \\
\hline La Rioja & & & & & 0 \\
\hline Santiago del Estero & & & & & 0 \\
\hline Sin datos & & 9 & 3 & 1 & 13 \\
\hline
\end{tabular}

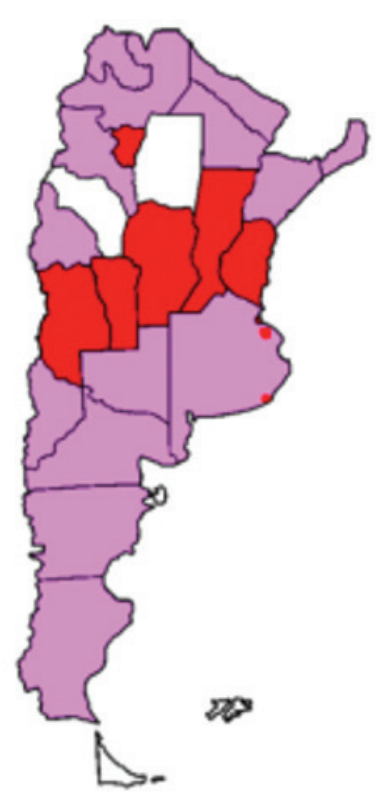

Figura 1. Distribución relativa de los aportes sobre salud en los congresos de EU en relación a las universidades por provincia: más oscuro más aportes 


\section{Red bibliográfica utilizada en la elaboración de ponencias sobre salud en las ponencias de los congresos nacionales de extensión de argentina (1997-2010)}

Para esta cuestión se examinaron 1319 referencias bibliográficas correspondientes a 167 ponencias (o ponencias sin ellas) acotadas a la temática de salud (criterio de inclusión) de los congresos nacionales de extensión que brindan información sobre bibliografía (El congreso de Cuyo 1997 no se ha considerado para este aspecto porque posee libro de resúmenes y no de ponencias lo que explica 167 trabajos en lugar de los 192 consignados en la tabla I) que se codificaron con un número único de identificación. Los criterios que se usaron para clasificar las ponencias fueron: 1) Ponencias con bibliografía no vinculada a extensión universitaria o su campo semántico y/o asociativo; 2) Ponencias con bibliografía vinculada directamente a extensión universitaria o su campo semántico y y/o asociativo; 3) Ponencias con bibliografía vinculada indirectamente a extensión universitaria o su campo semántico y/o asociativo; 4) Ponencias que no poseen bibliografía.

Aplicando el subprograma NetDraw.2086 de UCINET se obtuvo el grafo de la Figura 2 en donde se muestran los vínculos entre ponencias relacionadas por bibliografía común en los tres congresos.

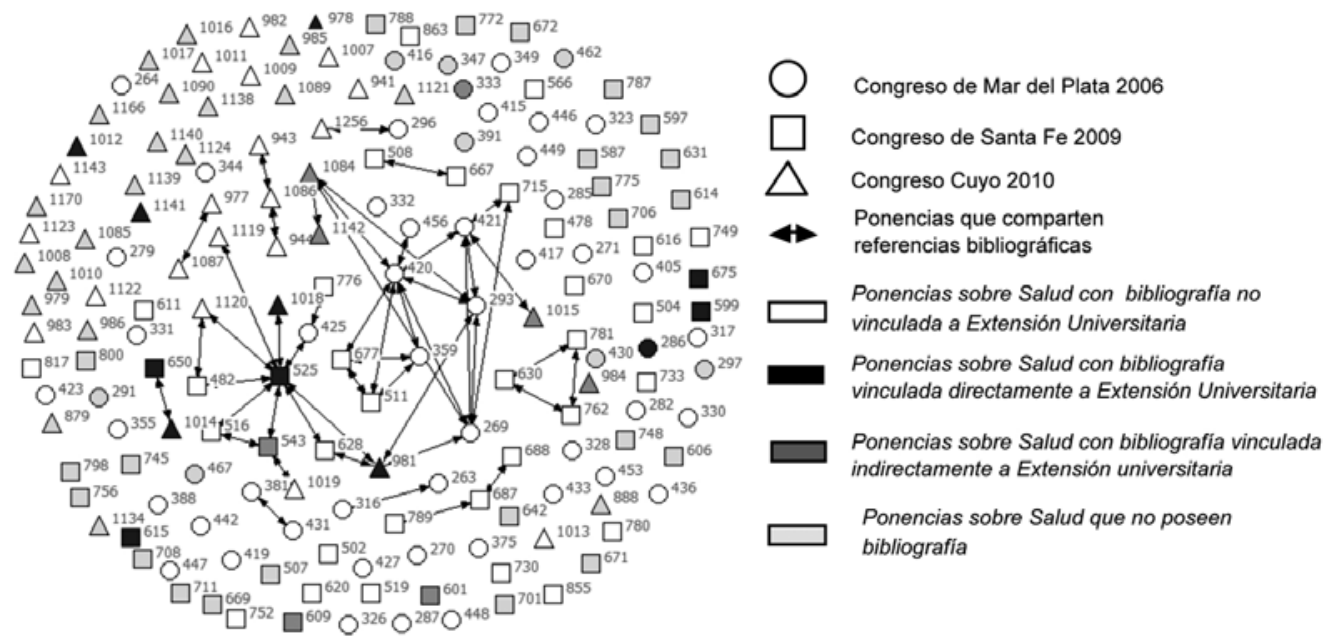

Figura 2. Red bibliográfica en el área de salud de los congresos nacionales de extensión universitaria de Argentina (1997-2010)

\section{Elección de objetos, sujetos y áreas temáticas que se vienen priorizando en el discurso extensionista sobre salud en Argentina}

Los datos se organizaron en dos tablas sobre los siguientes ejes: 1) Los sujetos seleccionados para las intervenciones (Tabla 3); 2) Los objetos de intervención recortados dentro de la temática amplia de salud/salud pública (Tabla 4). 
Tabla 3

Sujetos abordados en el área de salud en los congresos nacionales de extensión universitaria de Argentina (1997-2010)

\begin{tabular}{|c|c|c|c|c|c|c|}
\hline \multirow{2}{*}{\multicolumn{2}{|c|}{$\begin{array}{c}\text { Sujetos de la extensión en salud } \\
\text { identificados }\end{array}$}} & \multicolumn{5}{|c|}{ Cantidad de trabajos } \\
\hline & & \multirow{2}{*}{$\begin{array}{c}\text { Cuyo } \\
1997 \\
3 \\
\end{array}$} & \multirow{2}{*}{$\begin{array}{c}\begin{array}{c}\text { M.d.P. } \\
2006\end{array} \\
6\end{array}$} & \multirow{2}{*}{$\begin{array}{c}\begin{array}{c}\text { Sta. Fe } \\
\mathbf{2 0 0 9}\end{array} \\
12 \\
\end{array}$} & \multirow{2}{*}{$\begin{array}{c}\begin{array}{c}\text { Cuyo } \\
2010\end{array} \\
5 \\
\end{array}$} & \multirow{2}{*}{$\begin{array}{c}\text { Total } \\
26\end{array}$} \\
\hline Agentes del sist & na de salud & & & & & \\
\hline \multirow{6}{*}{ Comunidad } & educativa de escuelas & -- & -- & 3 & -- & 3 \\
\hline & general & 4 & 10 & 17 & 21 & 52 \\
\hline & terapéutica & -- & -- & -- & 1 & 1 \\
\hline & de riesgo & 2 & 5 & 4 & 1 & 12 \\
\hline & rural & -- & 2 & 2 & -- & 4 \\
\hline & $\begin{array}{l}\text { Vulnerable / periférica } \\
\text { / empobrecida }\end{array}$ & -- & 3 & 4 & 2 & 9 \\
\hline \multirow{6}{*}{ Grupos etarios } & Adolescentes & 3 & 4 & 3 & -- & 10 \\
\hline & Adultos mayores & 3 & -- & 1 & -- & 4 \\
\hline & Jóvenes & -- & -- & -- & 3 & 3 \\
\hline & Jóvenes y adultos & -- & -- & 2 & -- & 2 \\
\hline & Niñez & -- & 13 & 7 & 6 & 26 \\
\hline & Niñez y adolescencia & -- & 2 & 1 & 3 & 6 \\
\hline Docentes & & - & 2 & -- & 3 & 5 \\
\hline \multicolumn{2}{|c|}{ Docentes y padres } & -- & 1 & -- & -- & 1 \\
\hline \multicolumn{2}{|c|}{ Familia feriante } & -- & -- & -- & 1 & 1 \\
\hline \multicolumn{2}{|c|}{ Fuerzas de seguridad } & 1 & -- & -- & -- & 1 \\
\hline \multicolumn{2}{|c|}{ Mujeres } & 1 & 4 & 2 & 1 & 8 \\
\hline \multicolumn{2}{|l|}{ Padres } & -- & 1 & -- & -- & 1 \\
\hline \multicolumn{2}{|c|}{ Pueblos originarios } & 1 & -- & -- & -- & 1 \\
\hline \multicolumn{2}{|c|}{ Sistema de salud } & 2 & -- & 1 & 3 & 6 \\
\hline \multicolumn{2}{|l|}{ Trabajadores } & -- & 1 & 1 & -- & 2 \\
\hline \multicolumn{2}{|c|}{ Sin sujeto / sin referencia } & 2 & 1 & 3 & 2 & 8 \\
\hline
\end{tabular}

Tabla 4

Objetos abordados en el área de salud en los congresos nacionales de extensión universitaria de Argentina (1997-2010)

\begin{tabular}{lccccc}
\hline \multirow{2}{*}{$\begin{array}{c}\text { Objetos recortados dentro de la } \\
\text { temática de salud/salud pública }\end{array}$} & \begin{tabular}{c} 
Cuyo \\
\cline { 2 - 6 }
\end{tabular} & $\begin{array}{c}\text { M.d. P. } \\
\mathbf{2 0 9 7}\end{array}$ & $\begin{array}{c}\text { Sta. Fe } \\
\mathbf{2 0 0 6}\end{array}$ & $\begin{array}{c}\text { Cuyo } \\
\mathbf{2 0 1 0}\end{array}$ & Total \\
\hline Abordaje multidisciplinario en salud & 1 & & & & 1 \\
Aborto & & 1 & & & 1 \\
Adicciones & & 2 & 1 & 1 & 4 \\
Alimentación-nutrición & 2 & 7 & 6 & 1 & 16 \\
\hline
\end{tabular}




\begin{tabular}{|c|c|c|c|c|c|c|}
\hline \multirow{2}{*}{\multicolumn{2}{|c|}{$\begin{array}{l}\text { Objetos recortados dentro de la } \\
\text { temática de salud/salud pública }\end{array}$}} & \multicolumn{5}{|c|}{ Cantidad de trabajos } \\
\hline & & $\begin{array}{c}\text { Cuyo } \\
1997\end{array}$ & $\begin{array}{l}\text { M.d. P. } \\
2006\end{array}$ & $\begin{array}{c}\text { Sta. Fe } \\
2009\end{array}$ & $\begin{array}{l}\text { Cuyo } \\
2010\end{array}$ & Total \\
\hline \multicolumn{2}{|c|}{ Animales venenosos } & & 1 & & & 1 \\
\hline \multicolumn{2}{|c|}{ Cáncer } & 2 & 1 & 1 & & 4 \\
\hline \multicolumn{2}{|c|}{ Citología Hemática } & 1 & & & & 1 \\
\hline \multicolumn{2}{|c|}{ Cuidadores de enfermos } & & & 1 & & 1 \\
\hline \multicolumn{2}{|c|}{ Diagnóstico en salud } & 1 & & & & 1 \\
\hline \multicolumn{2}{|l|}{ Discapacidad } & 1 & 3 & 2 & & 6 \\
\hline \multicolumn{2}{|c|}{ Donación de órganos } & & & 1 & & 1 \\
\hline \multicolumn{2}{|c|}{ Donación de sangre } & & & 1 & 1 & 2 \\
\hline \multicolumn{2}{|c|}{ Educación sanitaria } & & & 1 & & 1 \\
\hline \multirow{4}{*}{ Enfermedades } & crónicas & & 3 & 1 & 4 & 8 \\
\hline & infecciosas & 1 & 1 & 1 & & 3 \\
\hline & vectoriales & & & 4 & 2 & 6 \\
\hline & zoonóticas & & & 1 & & 1 \\
\hline \multicolumn{2}{|c|}{ Estadística en salud } & 1 & & 1 & & 2 \\
\hline \multicolumn{2}{|c|}{$\begin{array}{l}\text { Experiencias de articulación entre cen- } \\
\text { tros de salud }\end{array}$} & & 1 & & & 1 \\
\hline \multicolumn{2}{|c|}{ Farmacia } & & & 1 & & 1 \\
\hline \multicolumn{2}{|c|}{ Fisiología Humana } & & & 1 & & 1 \\
\hline \multicolumn{2}{|c|}{ Fitomedicinas } & & 2 & 2 & 2 & 6 \\
\hline \multicolumn{2}{|l|}{ Fracaso escolar } & 1 & & & & 1 \\
\hline \multicolumn{2}{|c|}{ Herramienta de comunicación en salud } & 1 & & & & 1 \\
\hline \multicolumn{2}{|c|}{ Higiene } & & 1 & & & 1 \\
\hline \multicolumn{2}{|c|}{ Informática en salud } & & & & 1 & 1 \\
\hline \multicolumn{2}{|c|}{ Investigaciones sobre salud } & & & 1 & & 1 \\
\hline \multicolumn{2}{|c|}{ Líderes comunitarios en salud } & 1 & & & & 1 \\
\hline \multicolumn{2}{|c|}{ Maternidad } & 1 & 3 & 2 & & 6 \\
\hline \multicolumn{2}{|l|}{ Medicamentos } & 1 & & 1 & & 2 \\
\hline Parasitosis & & & 3 & 1 & & 4 \\
\hline Patología psico & social & & 1 & & & 1 \\
\hline Plaguicidas & & & & & 1 & 1 \\
\hline Primeros auxili & & 1 & 1 & & & 2 \\
\hline Relación Unive & rsidad-Servicios de salud & 1 & & & & 1 \\
\hline & auditiva-lenguaje & & 4 & 1 & 1 & 6 \\
\hline & bucal & 1 & & 8 & 15 & 24 \\
\hline & cardiovascular & & 1 & 1 & & 2 \\
\hline Salud & comunidades rurales & & 1 & & & 1 \\
\hline salua & en general & 1 & 9 & 8 & 6 & 24 \\
\hline & escolar & & & 1 & & 1 \\
\hline & laboral & & & 1 & 1 & 2 \\
\hline & mental & & 1 & 2 & 3 & 6 \\
\hline
\end{tabular}




\begin{tabular}{|c|c|c|c|c|c|c|}
\hline \multirow{2}{*}{\multicolumn{2}{|c|}{$\begin{array}{l}\text { Objetos recortados dentro de la } \\
\text { temática de salud/salud pública }\end{array}$}} & \multicolumn{5}{|c|}{ Cantidad de trabajos } \\
\hline & & $\begin{array}{l}\text { Cuyo } \\
1997\end{array}$ & $\begin{array}{l}\text { M.d. P. } \\
2006\end{array}$ & $\begin{array}{c}\text { Sta. Fe } \\
2009\end{array}$ & $\begin{array}{c}\text { Cuyo } \\
2010\end{array}$ & Total \\
\hline \multirow{4}{*}{ Salud } & sexual y reproductiva & 2 & 1 & & 3 & 6 \\
\hline & visual & & & 2 & 2 & 4 \\
\hline & y agua & & 1 & & & 1 \\
\hline & y medioambiente & & & 1 & 1 & 2 \\
\hline \multicolumn{2}{|c|}{ Servicios de salud } & & & 1 & 1 & 2 \\
\hline \multicolumn{2}{|l|}{ Strés } & & 1 & & & 1 \\
\hline \multicolumn{2}{|c|}{ Terapias y actividades con animales } & & & & 1 & 1 \\
\hline \multicolumn{2}{|c|}{ Tercera edad } & 3 & 2 & 1 & 1 & 7 \\
\hline \multicolumn{2}{|c|}{ VIH/SIDA } & & & 1 & & 1 \\
\hline \multicolumn{2}{|c|}{ Zoonosis } & & & & 2 & 2 \\
\hline \multicolumn{2}{|c|}{ Sin referencia } & 1 & 2 & 3 & 1 & 7 \\
\hline
\end{tabular}

\section{Discusión}

Para el primer aspecto analizado, observando la Figura 2 se pueden establecer algunas medidas propias del ARS (Menéndez, 2003):

- De un total de 167 ponencias, 45 (27\%) del total, comparten bibliografía.

- La red se compone de una red central, 9 islas y 122 ponencias aisladas (no vinculadas o que no comparten bibliografía).

- La densidad de la red aplicando la fórmula densidad=2L / n (n-1) y reemplazando:

Den= 2 x 48 Líneas o vinculaciones / 167 nodos $(167$ nodos -1$)=0,0034$.

- Las ponencias con mayor centralidad e intermediación son: 525 (nodo de grado 9); 432 (grado 8); 281 (grado 6); 433 (grado 5); 305, 669, 991 y 1086.

- Se detectaron dos camarillas o cliques en el Congreso de Santa Fe que corresponden a grupos de ponencias de un mismo autor o grupo de autores que comparten la misma base bibliográfica (ponencias: 630-762-781 y 482-525).

- Por otra parte se puede derivar del grafo el siguiente cuadro distributivo de ponencias reagrupadas por congreso y en relación a los criterios de clasificación (Tabla 5).

El análisis de bibliografía compartida busca establecer que obras están teniendo un posible carácter orientativo en la elaboración de una praxis extensionista y como se refleja esto en términos de categorías acerca de la extensión universitaria que emergen de las ponencias. En relación a los trabajos de los congresos sobre el tema salud el examen del grafo y la Tabla 5 muestran que la densidad de bibliografía compartida es sumamente baja. Cuando se considera al eje extensión universitaria el soporte bibliográfico con que cuentan los trabajos es más escaso aún lo cual no 
Tabla 5

Composición de la bibliografía para el área salud en los Congresos Nacionales de Extensión Universitaria de Argentina (1997-2010) en relación al objeto Extensión Universitaria.

\begin{tabular}{lcccc}
\hline & $\begin{array}{c}\text { Mar del } \\
\text { Plata 2006 }\end{array}$ & $\begin{array}{c}\text { Santa Fe } \\
\text { 2009 }\end{array}$ & Cuyo 2010 & Total \\
\hline $\begin{array}{l}\text { Ponencias con bibliografía no vinculada a } \\
\begin{array}{l}\text { Extensión Universitaria o su campo semán- } \\
\text { tico y y/o asociativo. }\end{array}\end{array}$ & 45 & 32 & 19 & 96 \\
$\begin{array}{l}\text { Ponencias con bibliografía vinculada direc- } \\
\text { tamente a Extensión universitaria o su cam- } \\
\text { po semántico y y/o asociativo. }\end{array}$ & 1 & 5 & 6 & 12 \\
$\begin{array}{l}\text { Ponencias con bibliografía vinculada indi- } \\
\text { rectamente a Extensión universitaria o su } \\
\text { campo semántico y y/o asociativo. }\end{array}$ & 1 & 3 & 4 & 8 \\
Ponencias sin bibliografía. & 8 & 23 & 20 & 51 \\
\hline
\end{tabular}

sorprende al observar que casi un tercio de los mismos carecen absolutamente de referenciación. Si bien se revela una progresión en el uso de bibliografía desde el congreso de Mar del Plata al de Santa Fe (se observa una retracción en Cuyo 2010) se debe remarcar que esto es para el campo de la salud puesto que en otros trabajos (López, 2011, 2012), se pudo relevar que en los congresos de Mar del Plata 2006 y Cuyo 2010, tomando el conjunto de las temáticas de Extensión, se pasa de un 10\% a un $19 \%$ respectivamente de las ponencias que cuentan con apoyo bibliográfico sobre la temática extensionista. Determinadas entonces cuales son las ponencias clave sobre salud que cuentan con bibliografía sobre extensión universitaria (Figura 2 , ponencias $286,525,599,615,650,675,978,981,1012,1014,1018,1141$ ) una de las redundancias notables es la necesidad/deseo de desarrollar la acción extensionista desde una organización más allá de: la disciplina de origen de la carrera, la cátedra o la especialidad de quienes se desempeñan en ella. Probablemente la toma de conciencia sobre la extensión universitaria que provee el sustento bibliográfico en esta materia muestra que la complejidad de los problemas sociales requiere de la mirada de diferentes especialistas. Así se puede observar la reiteración de abordajes multidisplinarios, interdisciplinarios e incluso transdiciplinarios que suponen respectivamente la inclusión tanto en el diseño, el examen o las acciones del aporte de distintas profesionalidades: de manera independiente (multidisciplina); mediante la discusión en la que intervienen distintas profesionalidades en cada una de estas etapas (interdisicplina); hasta la posibilidad de que miembros de los equipos con representaciones específicas, de ciertos aspectos del conjunto de las especialidades que intervienen en un problema, puedan asumir el comando completo en su resolución (transdiciplina) (Motta, 2002).En todos los casos el objetivo es superar la visión parcial que ofrece la disciplina y mejorar el impacto de la respuesta. Las pro- 
fesiones que se articulan en el campo de la salud para estas acciones extensionistas son alternativamente las de: bioquímicos, médicos, trabajadores sociales, psicólogos enfermeros, nutricionistas y odontólogos. Las ponencias informan que ello estimula no sólo la acción interpersonal sino además interinstitucional e intrauniversitaria es decir la colaboración entre cátedras, facultades y miembros de los distintos claustros (docentes, alumnos, egresados y no docentes).

Por otra parte por las características del área de la salud, las prácticas de extensión encuentran la alternativa de instrumentarse como prácticas pre-profesionales de tipo asistencial u otras, en que, sin embargo, no ocurre como en otras profesiones colegiadas (por lo menos no se desprende de las ponencias analizadas), en que las actividades de extensión son visualizadas por los colectivos profesionales-colegios y/o asociaciones-como una invasión al campo laboral sobre todo de nóveles egresados (López, 2011).

En relación a los conceptos de extensión que aparecen en las ponencias en el campo de la salud como orientadoras de la práctica aparecen una serie de claves como:

- Considerar a la Extensión Universitaria como impulsor de la necesidad de conocer la realidad local para lograr una mejor vinculación e integración universidadsociedad y dar respuestas apropiadas y transformadoras;

- Aparece como referente del espíritu la Reforma Universitaria Argentina del 1918;

- La extensión universitaria se considera como función social de la universidad y uno de los factores centrales que aporta la misma al cambio social -en este caso en materia de salud- en contextos de injusticia y desigualdad para aportar a una mejor calidad de vida. En este sentido la extensión universitaria se visualiza como un servicio a la comunidad y una herramienta para enfrentar los aspectos negativos de la globalización donde se asigna protagonismo en esta vinculación a los sectores populares. Como una subcategoría dentro de las definiciones de Extensión Universitaria, también en la temática de la salud, aparece una reiteración que se observa en otras áreas temáticas de la Extensión Universitaria que es la recurrencia de vincular a la misma con docencia e investigación, algo bastante frecuente en el discurso universitario cuando el mismo se refiere a la función extensionista aunque parece ser un rasgo menos frecuente cuando este mismo discurso parte de las otras funciones de docencia o investigación que en principio parecen satisfacerse en sí mismas y requieren menos de esta trilogía o de evocaciones como parte de los usos y costumbres universitarios.

En relación al segundo aspecto (tablas 3 y 4 del apartado RESULTADOS) se puede decir que en el siglo XX se han identificado como productores fundamentales del discurso sobre los problemas sociales en Argentina, entre los cuales se cuenta la problemática de salud/salud pública que tiene protagonismo en este trabajo, a la Iglesia, el Sindicalismo y el Estado (Carballeda, 2006). A partir del material empírico presentado se fortalece el supuesto acerca del papel creciente que en materia de salud/salud pública adquiere el estado sobre fines del milenio, a partir de su sistema universitario y la función extensionista, y por ende sobre los problemas sociales. Es 
necesario hacer notar que si bien el sistema universitario argentino se compone de un subsistema estatal y otro privado este último no ha tenido incidencia en el discurso sobre salud desde la función extensionista según el relevamiento realizado.

En los 14 años que se desarrollaron los primeros cuatro Congresos Nacionales de Extensión Universitaria, si se amplía el universo adicionando los pósters a las ponencias de los congresos, 276 trabajos de un total de 1562 (17,7\%), estuvieron vinculados a salud/salud pública lo que no es un dato menor respecto a la importancia asignada al área si se considera la enorme cantidad de temáticas que se abordan en dichos eventos.

Desde el punto de vista de los organizadores propios de la salud/salud publica también se pudo constatar que el discurso extensionista, medido en cantidad de ponencias y posters, se concentra mayoritariamente en lo que es atención primaria de la salud (180 trabajos: $65 \%$ ), procesos formativos intrauniversitarios como capacitaciones, perfeccionamientos y actualizaciones (38 trabajos: 14\%) combinaciones de los niveles primario, secundario y terciario de atención (22 trabajos: $8 \%$ ), más lejanamente estudios (9 trabajos: $3 \%$ ), divulgación (6 trabajos: $2 \%$ ), solo atención secundaria de la salud(5 trabajos: 2\%), solo atención terciaria ( 5 trabajos: $2 \%$ ) y organización de los servicios de salud u otros temas (4 trabajos: menos del 1\%), sin referencias ( 3 trabajos: 1\%), otros ( 2 trabajos: $1 \%$ ).

Si bien uno de los analizadores de las problemáticas sociales y de la problemática en salud pública es la fragmentación social esta es producida tanto a nivel de la realidad como de la producción discursiva que la retroalimenta. Se puede observar que en los congresos de extensión existe una diversidad de sujetos que se pueden reagrupar, en: Grupos etarios (niñez, adolescencia, jóvenes, adultos y adultos mayores), comunidad/ población (en general, rural, escolar, de riesgo, vulnerable, terapéutica, de barrios periféricos), los agentes del sistema de salud (estudiantes, técnicos y profesionales), docentes de otros niveles educativos y el componente de género (las mujeres). Una ausencia significativa es el sujeto familia. Si bien la familia aparece todo el tiempo es más bien vinculada a unidades menores como los niños o mayores como la comunidad aunque poco como destinataria de las extensiones en los trabajos analizados donde es aludida más bien como comunidad de riesgo es decir como grupo cuya dinámica se puede ver afectada por la presencia o reinserción de pacientes con algún nivel de afectación en su salud (Tabla 3).

Por otra parte los objetos abordados, como surge de la Tabla 5, presentan una gran diversidad aunque los aspectos más atendidos desde la relación teoría práctica extensionista en salud/salud pública, según lo evidencian los Congresos Nacionales a través de los años, son la salud bucal, la alimentación/nutrición, la salud en general, las enfermedades vectoriales y la salud auditiva/lenguaje (Tabla 4). Llama la atención la escasa construcción de algunos objetos que han tenido un gran protagonismo en el discurso público de la salud en las últimas décadas tales como el VIH/SIDA y las adicciones (tabaquismo, droga, alcohol) por lo que es posible entonces que el discurso universitario extensionista se haya replegado en algunos nichos dejando el lugar a otros actores/agencias que tradicionalmente vienen trabajando estos temas como los organismos de salud pública, el sistema educativo, las iglesias y los sindicatos que conservan el rol y cierto monopolio instalado en materia de problemas sociales como se aludía al principio del parágrafo. 


\section{Conclusiones}

La red bibliográfica de las ponencias de los congresos nacionales de extensión de Argentina muestra que en el área salud la extensión universitaria se construye fundamentalmente con orientación bibliográfica propia del campo de la salud y muy escasamente proveniente de la extensión universitaria. Por otra parte, en la elección y formulación de sujetos y objetos de la extensión universitaria en materia de salud pública las intervenciones muestran una concepción progresista que intenta ir más allá del modelo de medicalización que se desarrolla en la región desde hace más de una centuria (Armus, 2005). Por un lado el organizador salud y otros tienen preeminencia respecto del organizador enfermedad(es) que acapara sólo el 11\% de las producciones (30 presentaciones entre ponencias y pósters relevados). Por otro lado los trabajos más que enfocarse en el individuo-paciente y en factores biomédicos desde una concepción monocausal o aun multicausal integran el contexto y los procesos histórico-territoriales con tendencia a ubicar los problemas de salud dentro de relaciones sociales más amplias por lo cual no es extraña la recurrente remisión al concepto de comunidad como principal destinatario. Es en la particular articulación entre docencia, investigación y extensión -predominante en la búsqueda de una identidad universitaria- que se producen procesos reflexivos que enriquecen los ámbitos de actuación sean estos los de la salud u otros.

\section{Referencias}

Armus, D. (2005). Avatares de la medicalización en América latina 1870-1970. Buenos Aires: Lugar.

Bourdieu, P. (2002). Campo de poder, campo intelectual. Buenos Aires: Montresor.

Bueno Campos, E. (2007). La tercera misión de la universidad: el reto del conocimiento. La universidad del futuro. Tribuna de debate, 41. Recuperado de http://www.madrimasd.org/revista/revista41/tribuna/tribuna.asp

Carballeda, A. (2006). El trabajo social desde una mirada histórica centrada en la intervención. Buenos Aires: Espacio.

García Guadilla, C. (1996). La educación superior como objeto de investigación: el caso de América Latina. En P. Krotsch, M.C. Nosiglia y O. Pisani (Comps.), Primer Encuentro Nacional La universidad como objeto de investigación. Buenos Aires: Oficina de Publicaciones del CBC, UBA.

López, M.L. (2005). Extensión en el Nivel Superior. Experiencia desarrollada en la FHYCSUNJU. Jujuy-Argentina: EdiUnju.

López, M.L. (2010). University Extension. Problem Identification and Guidance for Managing the Area. Revista Universidad y Sociedad del Conocimiento, 38(3), 1-8. Recuperado de http://rusc.uoc.edu/ojs/index.php/rusc/article/view/v7n2-lopez/v7n2lopez-eng

López, M.L. (2011). Extensión Universitaria aportes para su problematización a partir de la exploración bibliográfica. Revista de Estudios Sociales,7(2). 191-199. Recuperado de http://148.202.18.157/sitios/publicacionesite/pperiod/estusoc/articulos2.pdf

López, M.L. (2012). Extensión Universitaria situación actual y aportes metodológicos. JujuyArgentina: EdiUNJu. 
Maz-Machado, A., Bracho-López, R., Torralbo-Rodríguez, M., Gutiérrez-Arenas, M.P., Jiménez-Fanju, N. y Adamuz-Povedan, N. (2012). Redes académicas generadas por las tesis doctorales de educación matemática en España. Revista de Investigación Educativa, 30(2). 271-286. Recuperado de http://revistas.um.es/rie/article/view/ rie.30.2.116421/148841

Marimon, M.E. (2007). La extensión universitaria como vía para fortalecer los vínculos universidad-sociedad desde la promoción de salud. Revista Cubana de Salud Pública, 33(2), 1-10.

Menéndez, L.S. (2003). Análisis de redes sociales: o cómo representar las estructuras sociales subyacentes. Revista Apuntes de Ciencia y Tecnología, 7(1), 21-29.

Motta, R. (2002). Complejidad, educación y transdisciplinariedad, 3(1), 121. Recuperado de http://www.redalyc.org/articulo.oa?id=30510313

Pérez Martínez, V.T. (2010). Conocimientos sobre el proceso de extensión universitaria en un área de salud. Revista Cubana de Medicina General Integral, 26(1), 107-116. Recuperado de http://scielo.sld.cu/scielo.php?script=sci_arttext\&p $\mathrm{id}=$ S0864-21252010000100012

Soneira, A. J. (2007). La teoría fundamentada en los datos (Grounded Theory) de Glaser y Strauss. En I. Vasilachis (Ed.), Estrategias de Investigación Cualitativa (pp. 153-174). Buenos Aires: Gedisa.

Universidad Nacional de Cuyo (Ed.). (1997). I Congreso Nacional de Extensión de la Educación Superior. II Encuentro Latinoamericano de Extensión Universitaria. Cuyo: EdiUNCu.

Universidad Nacional de Mar del Plata (Ed.). (2006). II Congreso Nacional de Extensión "Un Intercambio con la Comunidad". Mar del Plata-Argentina: Ediciones Suárez.

Universidad Nacional del Litoral (Ed.). (2009). III Congreso Nacional de Extensión Universitaria "La integración, extensión, docencia e investigación. Desafíos para el Desarrollo Social". Santa Fe: Editorial de la UNL.

Universidad Nacional de Cuyo (Ed.). (2010). IV Congreso Nacional de Extensión Universitaria y IX Jornadas Nacionales e Extensión Universitaria "Compromiso social y calidad educativa: desafíos de la Extensión". Mendoza-Argentina: EdiUNCu.

Vázquez García, J.A. (2015). Nuevos escenarios y tendencias universitarias. Revista de Investigación Educativa, 33(1), 13-26. Doi: http://dx.doi.org/10.6018/rie.33.1.211501

Veliz Gutiérrez, J.A., Pérez Díaz, N., Fernández Montenquín, Z., Véliz Martínez, D., Pérez, N.S.C. (2011). La extensión universitaria y la promoción de salud en la atención primaria. Revista Ciencias Médicas de la Universidad Médica de Pinar del Río, 15(4), 218-230.

Fecha de recepción: 22/12/2014

Fecha de revisión: 23/12/2014

Fecha de aceptación: 04/06/2015 